\title{
Clinical management of inflammatory bowel disease: Beyond disease activity. Part II: Strategies for maximizing psychosocial health
}

\author{
T Michael Vallis, PhD, GeofFrey K Turnbull, MD
}

TM VALLIS, GK TURNBULL. Clinical management of inflammatory bowel disease: Beyond disease activity. Part II: Strategies for maximizing psychosocial health. Can J Gastroenterol 1992;6(2):87-92. Inflammatory bowel disease (IBD) remains a chronic, relapsing disorder that can be very disabling to the patient and often leads to significant problems in living (eg, emotional distress, social isolation, work impairment and disability). In Part I of this paper, published in an earlier issue of this Journal, the authors reviewed available evidence indicating that health status is influenced strongly by psychosocial factors in addition to disease activity. The purpose of the present paper is to provide a specific framework to guide the gastroenterologist in the assessment and management of psychosocial factors that impact on the health status of the IBD patient. Guidelines for managing these psychosocial factors are provided. A central thesis of this paper is that the gastroenterologist, whether alone or in conjunction with a mental health specialist, must manage psychosocial as well as disease activity factors in the ongoing care of patients. (Pour résumé, voir page 88)

Key Words: Inflammatory bowel disease, Psychosocial problems, Psychosocial treatment, Quality of life

Departments of Psychology, Medicine and Gastroenterology, Camp Hill Medical Centre and Dalhousie University, Halifax, Nova Scotia

Correspondence and reprints: Dr TM Vallis, Department of Psychology, Camp Hill Medical Centre, 1763 Robie Street, Halifax, Nova Scotia B3H 3 G2

Received for publication August 6, 1991. Accepted January 31, 1992
IN PART 1 OF THIS TWO-PART SERIES, the authors reviewed the literature on the role of psychosocial factors in inflammatory bowel disease (IBD) (1). This review indicated that it is inappropriate, based on the accumulated scientific data, to view IBD as due to psychopathology (eg, the 'Crohn's personality' as causal). There is support, however, for the notion that IBD can result in psychiatric difficulties, but the majority of IBD patients does not meet the criteria for a psychiatric diagnosis. Therefore, the most valuable approach to evaluating psychosocial factors in IBD is to adopt a quality of life perspective from which psychosocial difficulties are seen as the result, not the cause, of IBD. The authors' previous review further indicated that IBD (particularly Crohn's disease), negatively impacts on a variety of psychosocial factors including emotional distress, feelings of well- 
Traitement clinique du syndrome du côlon irritable: Au-delà de l'activité de la maladie. Partie II: Stratégie de maximisation du bien-être psychosocial

RESUME: Le syndrome du côlon irritable (SCI) est une affection chronique et récidivante qui peut être invalidante et perturbe souvent le mode de vie (troubles émotionnels, isolement social, incapacité de travail et invalidité). Dans la partie I du présent article publié précédemment, les auteurs examinaient les observations indiquant que, en plus de l'activité de la maladie, l'état de santé du patient est fortement influencé par des facteurs psychosociaux. Ces travaux visent à fournir un cadre de travail qui guidera le gastroentérologue dans l'évaluation et la gestion des facteurs psychosociaux qui influent sur l'état de santé du patient porteur du SCI. Ils fournissent des directives permettant de gérer ces facteurs. La thèse centrale des auteurs est que le gastroentérologue, seul ou avec la collaboration d'un spécialiste de la santé mentale, doit continuer à traiter à la fois les aspects psychosociaux et les facteurs d'activité de la maladie chez ses patients.

being, interpersonal relationships and symptom management (eg, physician visits). Given the complexity involved in the impact of IBD on psychosocial factors, an assessment that incorporates increasingly specific levels of inquiry is recommended. The current study illustrates this by describing in detail a sequenced approach to assessment and a treatmene approach that can be followed by the gastroenterologist.

\section{STRATEGIES FOR MAXIMIZING PSYCHOSOCIAL HEALTH IN IBD}

It should be noted that disease activity cannot be treated separately from the psychosocial context of the IBD patient. Therefore, the gastroenterologist is faced with managing these psychosocial issues, either alone or in conjunction with other health care providers. The following is intended as

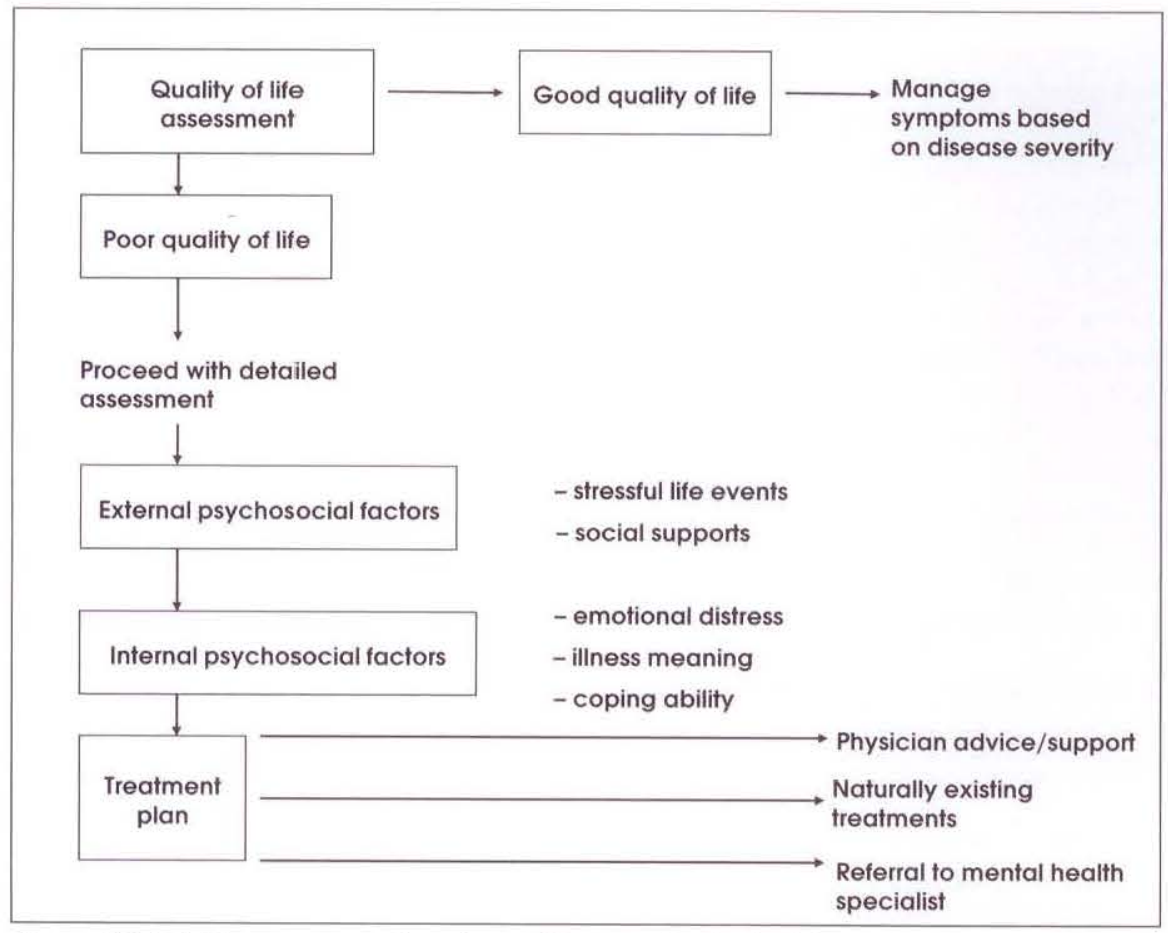

Figure 1) Model for assessment of psychosocial factors related to health status in inflammatory bowel disease a guide for the assessment of psychosocial factors, along with recommendations for treatment. Unless demonstrated otherwise (eg, in cases where IBD patients have clear psychiatric difficulties which may have preceded the development of IBD), it is best to assume that psychosocial problems related to IBD are the result, not the cause, of the disease process. To guide the reader, a schema of the authors' recommendations is provided in Figure 1.

Stage 1: The authors recommend that assessment of the IBD patient begin by evaluating overall quality of life. Different levels of formality can be used in this assessment, ranging from general questions concerning overall life satisfaction to more specific questions about coping with IBD and its impact, and possibly including detailed assessments with semistructured interviewing and/ or questionnaires. Attention should be paid to psychological well-being as well as psychological distress; both areas should be probed specifically. Gastroenterologists should become familiar with a quality of life measure - these questionnaires tend to be easy to administer and often can be completed quickly by patients in a waiting room. The questionnaires can be general quality of life measures, not specific to IBD, such as the sickness impact profile (2) or the mental health inventory (3). IBD-specific quality of life measures, such as the IBD questionnaire (4), are becoming available. It should be noted, however, that normative data will have to be collected on these instruments with IBD patients in order to interpret validly a given patient's responses.

Stage 2: Assessing overall quality of life will identify a subsample of patients requiring further assessment (those with poor well-being and/or emotional distress). For the remaining group of patients (ie, those whose quality of life is good), the gastroenterologist can manage the illness experience by focusing on disease activity variables alone. The management of those requiring further assessment likely will be more involved, however, depending on assessment results. In stage 2 assessment, the authors recommend that the gastroenterologist assess relevant psychosocial factors ex- 
ternal to the individual. Stressful events and social supports should be assessed.

When inquiring into stressful events attention should be paid to stressors associated with the disease (for example, stress experienced by a young woman because of weight gain due to steroid use) as well as to other types of stressors. Disease-specific stressors include a patient' s history of IBD relapses plus the extent and invasiveness of past and current medical treatments. Further, research on the role of stressful life events has demonstrated the importance of separating major life events (eg, divorce, unemployment, financial debt, death of a loved one) from minor daily hassles (such as commuting long distances in heavy traffic, minor disagreements with co-workers). These nondisease-specific stressors have been shown to impact negatively on quality of life and illness experience (5).

While stressful events can detract from a person's quality of life, social supports enhance it by exerting a positive influence in at least two ways (6): directly improving self-esteem and life satisfaction, and serving as a buffer, preventing negative factors, such as stressful events, from having a substantial impact. For example, recovery from surgery or adjustment to an ostomy bag can be improved by supportive family members. Lack of social support can magnify the stress associated with an illness, and it has been shown that support can predict illness onset (7). As well, it is common for the illness experience itself to lead to decreases in social support (eg, the middle-aged man with Crohn's disease who withdraws from social service clubs due to concern of embarrassing himself by incontinence).

It is recommended that the gastroenterologist inquire specifically into these aspects of a patient's life. Inquiry about general supports should be made (eg, "do you have people in your life that you can rely on when you need them?") as well as about support specifically related to IBD and its management (eg, "do you have people in your life who help you deal with your physical symptoms?"). Family composition, current living situation and resources for child care/family responsibilities are useful to assess, either as sources of support or psychosocial difficulty. If significant problems are uncovered, intervention into these areas should be incorporated into the management plan.

Stage 3: In addition to the external factors, the illness experience can be influenced strongly by internal factors. Illness meaning, emotional distress and coping ability/style should be examined.

Research into the psychology of illness consistently has demonstrated that patients' beliefs about their symptoms (cause and cure) are very powerful influences on their behaviour (8). Therefore, it is helpful to inquire into patients' specific appraisals of their illness. Research into this area suggests that individuals develop idiosyncratic beliefs about the identity, consequences, cause and time frame of their symptoms. By understanding the patient's own views on these issues (which often requires persistence in questioning), much valuable information can be gained. For instance, with the patient who believes his or her symptoms to be minor and of no consequence, lack of adherence to a medical regimen would not be surprising. Similarly, hopelessness can be understood if a patient views the illness as imminently life-threatening and uncontrollable (regardless of the actual disease severity or prognosis).

Several psychometric scales have been developed to assess systematically dimensions on which patients make judgements about illnesses. One such questionnaire, the implicit models of illness questionnaire, is composed of four subscales which assess seriousness, control, change and personal responsibility (9). The use of these scales, initially in research studies and eventually for clinical purposes, will improve one's ability to understand and intervene upon this important dimension.

Emotional distress is an important area of functioning for all individuals, and there is some suggestion that distress mediates help seeking (10). Three types of emotional distress particularly are important to explore: depression/ dysphoria, anxiety/worry and anger/ frustration.

In assessing the presence and intensity of these emotions one should not be restricted to psychiatric diagnostic conditions. Often with medical patients there is significant distress that does not involve the classic symptoms required for a psychiatric diagnosis. Further, distress might be mediated by the illness, either directly (eg, the individual who is depressed because of IBD symptoms) or indirectly (eg, the individual who is depressed because he or she cannot work due to IBD symptoms or because his or her spouse has left due to difficulty coping with IBD). Anger and frustration particularly are important to explore and often are overlooked. It needs to be appreciated that by virtue of having a chronic illness, physician visits are a necessary, but not always welcome, aspect of care for IBD patients. Adherence problems often are mediated by unexpressed or unacknowledged frustration at the medical system and its limits. Open discussion about inevitable frustrations can go a long way in circumventing such problems.

Research into coping ability/style indicates that there is a high degree of difference in how individuals handle stressful situations. Inquiring into typical strategies and their effectiveness is recommended. Distinguishing between active (information seeking, problem solving, etc) and passive (praying, for example) forms of coping can be useful. Asking for specific examples often provides sufficient detail to judge the adequacy of a person's coping ability. One must also take into consideration the degree of control the individual actually may have over the situation. There is greater active control associated with social isolation in response to disease diagnosis then there is with adjusting to the stress of an ostomy bag or forced retirement from work (ie, the latter are less easily reversed than the former).

Stage 4: By following the above assessment framework, the gastroenterologist implementing treatment strategies will develop a comprehensive understanding of the full illness experience of an IBD patient. There are complex presentations possible given the number of 
TABLE 1

Procedure for treating inflammatory bowel disease within the gastrointestinal unit, Camp Hill Medical Centre

Gastroenterologist assessment
Quality of life
(general)

Adjustment to disease

Overall level of distress

\section{Psychosocial adjustment \\ Resources}

Education

Employment

Supports

Family composition (nuclear and extended)

Living situation

Drug plan benefits

Stressors

Treatment

History of IBD flare-ups

Extent/invasiveness of past medical treatment

Difficulties with employment

General negative life experiences

Flexible use of in/outpatient services

Regular follow-up

Ongoing assessment of disease activity

Advice, support and encouragement
Psychologist assessment

Quality of life

(specific)

Emotional well-being (type and extent of coping)

Psychological distress

(presence of significant emotional difficulties)

Psychosocial adjustment

Resources

General coping ability: potential to improve coping

Potential for family to change

Supports

Psychological
assessment of
perceived/tangible
supports
Assess family and
support network

Stressors

Daily hassles

Major stressful life events

Assess skill at coping with stressors

Treatment

Stress management training

Individual

psychotherapy

Problem-solving training

Advice, support and encouragement

IBD Inflammatory bowel disease

factors and their interactions. Despite this complexity, the assessment task is important to tailor a specific treatment to meet the individual needs of the patient. The following approach to treatment is recommended by the current authors. The cornerstone of management strategy for the gastroenterologist should remain medical intervention based on disease activity. In addition, the principle of parsimony (if a choice between a simple and complex treat- ment exists, choose the simple when all else is equal) is advocated in planning management to add to treatment efficacy and appropriateness. Three main strategies should be considered.

First, attention should be focused on physician advice, education and support. Assessment of the psychosocial factors related to quality of life in IBD is an essential part of treatment. Given a clear and detailed understanding of disease activity as well as internal and external psychosocial factors, many issues which impair treatment can be dealt with efficiently by the gastroenterologist. Understanding, support, advice and education quickly will prove effective (11). However, without an awareness or understanding of relevant psychosocial factors, no such intervention can take place.

Second, in situations where intervention by the gastroenterologist is insufficient, or for some reason inappropriate, consideration should be given to naturally existing treatments or other forms of intervention before considering referral to other health care professionals (which often requires extensive waiting periods, and appropriate resources may not exist). Making use of family support systems (nuclear as well as extended family) and encouraging involvement in community organizations, either general (such as the YM/ WCA) or IBD-specific (eg, the Canadian Foundation for Ileitis and Colitis) can go a long way to overcome dysphoria, anxiety and general distress, and can improve social isolation and function.

In cases where office consultation and natural support systems are inadequate to address the needs of the IBD patient with impaired quality of life, referral to a mental health professional, the third strategy, should be considered. Referrals may be of two types. The preferred referral would be to a psychologist, psychiatrist or social worker with specific interests and expertise in IBD. If no specialized service exists, referral to a general mental health service is recommended. By following these treatment options in the sequence presented above (ie, moving to a more invasive treatment only once a less invasive treatment has failed), the greatest therapeutic benefit can be realized in the most cost effective manner.

Readers may be interested in the approach to management of $I B D$ patients within the gastrointestinal unit at $\mathrm{Dal}$ housie University's Camp Hill Medical Centre. A collaborative arrangement has been developed between gastroenterology and psychology to manage the psychosocial needs of IBD patients. Psychology runs a one-day clinic in the 
gastrointestinal unit to maximize communication between the disciplines. The psychologist serves as a consultant to gastroenterologists, either to discuss the management of a specific case or to assess and treat the patient directly. A general schema of the running of this service is presented in Table 1. Included are the types of inquiries made by the gastroenterologist and by the psychologist, and how they work together.

\section{CURRENT STATE OF PSYCHOLOGICAL TREATMENT OF IBD}

This paper would not be complete without a brief review of the effectiveness of psychosocial treatments for IBD. Currently there are very few psychosocial treatment studies published. Groen and Bastiaans (12) reported on 35 ulcerative colitis patients of whom 29 were treated almost exclusively with supportive psychotherapy. No reliable outcome measures were reported and no experimental methodology was employed. Nonetheless, the authors asserted that psychotherapy can be highly effective provided the therapist (physician or psychiatrist) has a genuine concern for patients. Freyberger et al (13) also reported on supportive psychotherapy (followed by dynamic interventions) in a small group of IBD patients. Using student therapists they demonstrated greater improvement on measures of mood (anxiety and depression) in a group of patients treated with psychotherapy versus a control group of untreated patients. No data on physical symptoms were reported, and the data were collected over just one week.

In contrast to a supportive or dynamic model of psychotherapy, Joachim and colleagues (14) evaluated the usefulness of stress management

\section{REFERENCES}

1. Vallis TM, Turnbull GK. Clinical management of IBD: Beyond disease activity. Part I: Assessing psychosocial factors. Can J Gastrenterol 1992;6:39-43.

2. Berger, M, Bobbitt RA, Pollard WE, Martin DP, Gilson DS. The sickness impact profile: Validation of a health status measure. Medical Care $1976 ; 14: 57-67$.

3. Veit CT, Ware JE. The structure of techniques with IBD patients. They treated a group of 15 patients with deep breathing, imagery and massage. No control group was used, but treatment was reported to promote feelings of well-being and increase coping with IBD. A randomized controlled trial of stress management was reported by Milne and co-workers (15). Eighty IBD patients were treated either with stress management (autogenics, problemsolving training and communication skills) or with a no-treatment control procedure. Measures of self-reported disease severity and psychosocial wellbeing were collected with results indicating that stress management led to significant improvement on both measures, whereas the control procedure did not (the two conditions were not compared directly with statistical procedures). Unfortunately, despite random assignment, the control group had significantly lower scores on symptom severity and distress measures prior to treatment. Therefore, lack of change in this group could be due to a floor effect.

In a recent study, Schwartz and Blanchard (16) reported on a controlled, randomized treatment study for a mixed sample of ulcerative colitis and Crohn's disease patients. Treatment consisted of education, stress management (relaxation training and biofeedback) and cognitive coping strategies.

A symptom-monitoring waiting list was the control group. Results on symptom measures indicated that treatment increased symptoms, relative to the control group, but treated subjects reported improvements on measures of distress and coping. Nonetheless, the data indicating that treatment had negative effects on symptom experience is alarming. Schwartz and Blanchard (16)

psychological distress and well-being in general populations. J Con Clin Psychol 1983;51:730-42.

4. Guyatt G, Mitchell A, Irvine EJ, et al A new measure of health status for clinical trials in inflammatory bowel disease. Gastroenterology 1989;96:804-10.

5. Brown GW, Harris TO. Life Events and Illness. New York: Guilford, 1989.

6. Cohen S, Syme SL. Issues in the Study have suggested that their treatment might be effective only for Crohn's disease patients. When they examined their data for ulcerative colitis and Crohn's disease patients separately only Crohn's disease patients showed positive responses. Clearly, future treatment studies will need to examine ulcerative colitis and Crohn's disease patients separately. Based on available data, psychosocial problems appear more prevalent with Crohn's disease compared with ulcerative colitis.

\section{CONCLUSIONS}

Based on the two papers in this series, it can be concluded that IBD, particularly Crohn's disease, can have a significant negative impact on quality of life. This effect occurs due to interaction of disease activity and associated psychosocial factors. It is best to view impaired quality of life linked with IBD as resulting from the illness. There is no clear evidence that reduced quality of life necessarily is due to psychopathology.

No evidence indicates that psychopathology causes IBD. A subsample of IBD patients will no doubt have psychiatric illness (either pre- or post dating the onset of illness), but it is unclear whether this occurs at a rate greater than in normal populations.

Treatment of the illness experience of IBD patients is organized best by the gastroenterologist who assesses general quality of life and (when appropriate) looks at relevant internal and external psychosocial factors, and then uses this assessment to plan treatment. Treatment should be approached flexibly and include regular medical follow-up, advice, support and education as well as naturally existing programs and referral to mental health specialists.

of Social Support and Health. New

York: Academic Press, 1985.

7. Zegans LS. Stress and the development of somatic disorders. In: Goldberg L,

Breznitz S, eds. Handbook of Stress:

Theoretical and Clinical Aspects.

New York: Free Press, 1983:134-52.

8. Leventhal $\mathrm{H}$, Zimmerman R,

Gutmann M. Compliance: A selfregulation perspective. In: Gentry WD, ed. Handbook of Behavioral Medicine. New York: Guilford, 1984:369-436. 
9. Turk DC, Rudy TE, Salovey P. Implicit models of illness. J Behav Med 1986;9:453-74.

10. McHugh S, Vallis TM. Illness Behaviour: A Multidisciplinary Model. New York: Plenum, 1986.

11. Kurlinder KA, O'Brien MF. Psychosocial issues. In: Peppercorn MA, ed. Therapy of Inflammatory Bowel Disease: New Medical and Surgical Approaches. New York: Dekker, 1990:243-65.
12. Groen J, Bastiaans J. Psychotherapy and ulcerative colitis.

Gastroenterology 1951;18:344-52.

13. Frayberger H, Kunsebeck H-W, Lempa W, Wellmann W, Avenarius H-J. Psychotherapeutic interventions in alexithymic patients: With special regard to ulcerative colitis and Crohn's patients. Psychother Psychosom 1985;44:72-81.

14. Joachim G. The effects of two stress management techniques on feelings of well-being in patients with inflammatory bowel disease. Nurs Pap 1983;15:15-8.

15. Milne B, Joachim G, Niedhardt ]. A stress management program for inflammatory bowel disease patients. J Advan Nurs 1986;11:561-7.

16. Schwarz SP, Blanchard EB. Evaluation of psychological treatment for inflammatory bowel disease. Behav Res Ther 1991;29:167-77. 


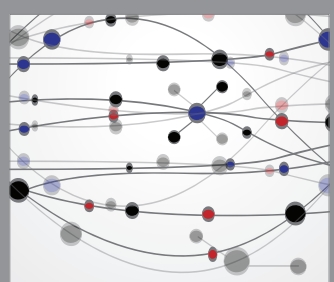

The Scientific World Journal
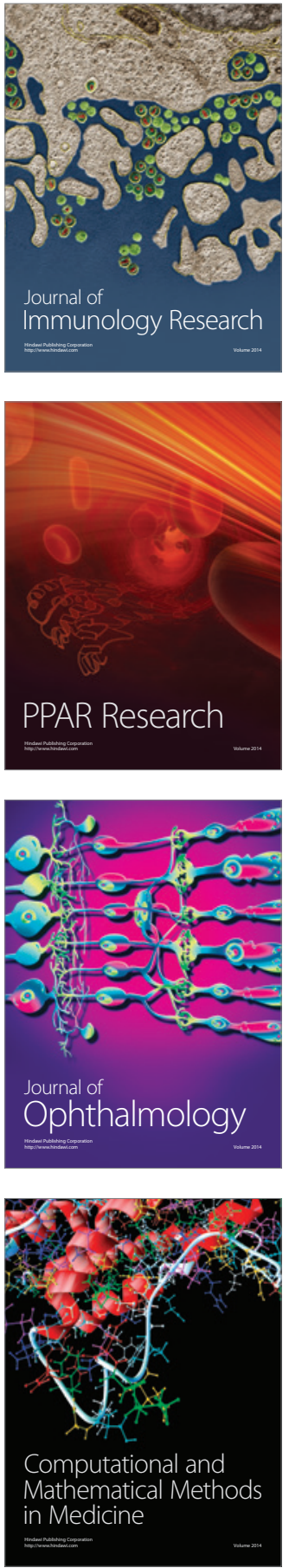

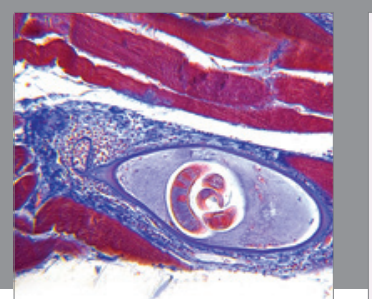

Gastroenterology Research and Practice

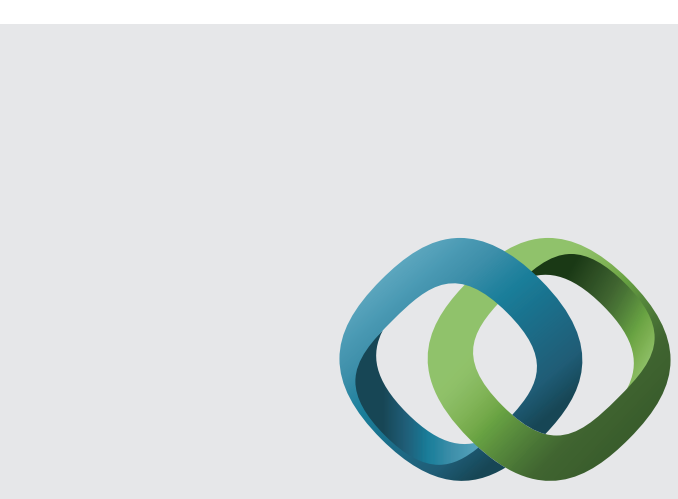

\section{Hindawi}

Submit your manuscripts at

http://www.hindawi.com
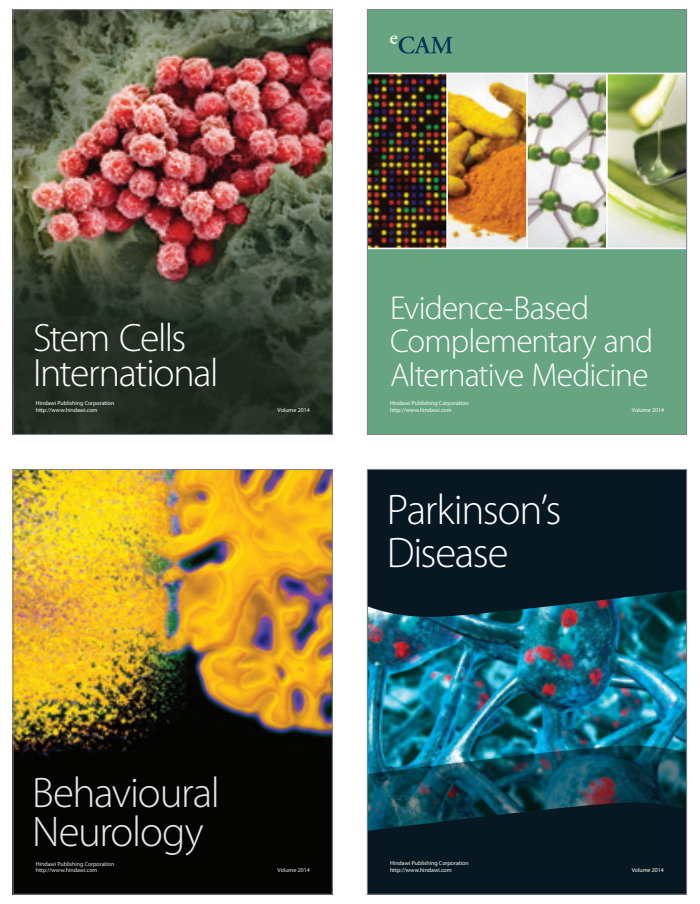
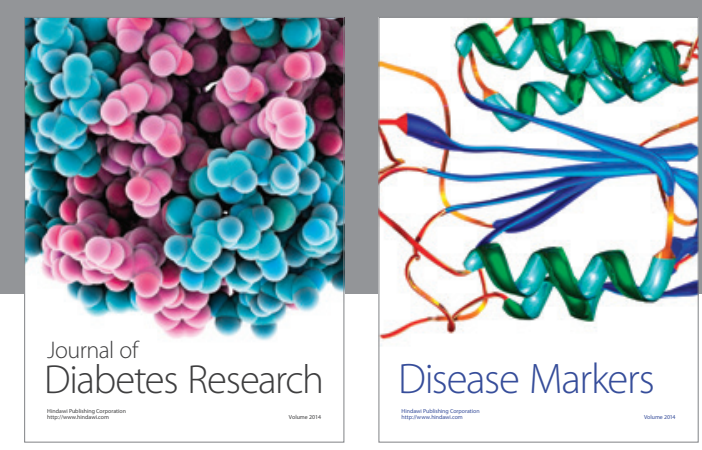

Disease Markers
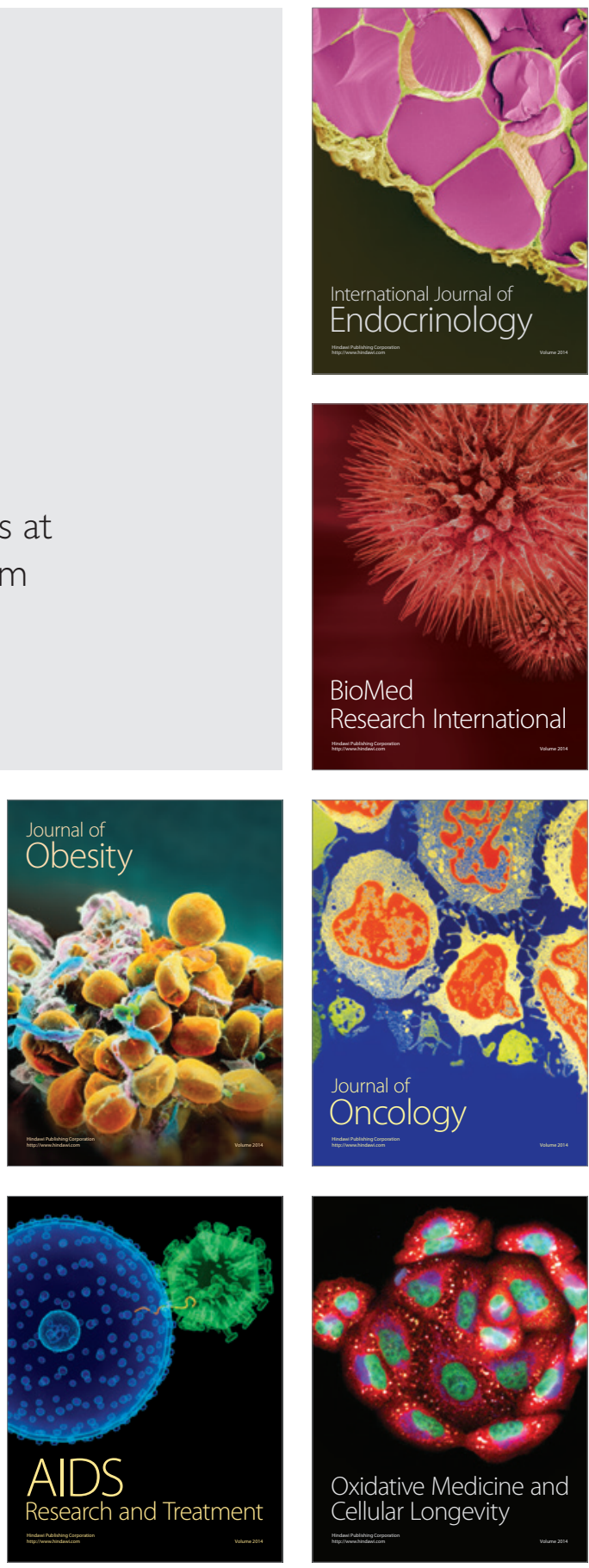